\title{
AVALIAÇÃO DE CULTIVARES E HÍBRIDOS DE BANANEIRA NO RECÔNCAVO BAIANO
}

\author{
Evaluation of banana cultivars and hybrids in northeastern Bahia
}

\author{
Marcelo Bezerra Lima ${ }^{1}$, Sebastião de Oliveira e Silva ${ }^{2}$, Onildo Nunes de Jesus ${ }^{3}$, \\ Waldete Souza Japiassu de Oliveira ${ }^{4}$, Marlon da Silva Garrido ${ }^{5}$, Ruberval Leone Azevedo ${ }^{5}$
}

\begin{abstract}
RESUMO
O programa de melhoramento genético de bananeira da Embrapa Mandioca e Fruticultura Tropical visa desenvolver e selecionar novas cultivares de bananeira com alta produtividade, bom sabor dos frutos e que apresentem resistência às principais doenças. Objetivou-se com este trabalho avaliar cultivares e híbridos de bananeira visando a identificação de genótipos superiores em produtividade e resistência a doenças. Foram avaliados os híbridos da cv. Gros Michel (Calipso, Bucaneiro e Ambrosia); da cv.Yangambi (YB42-21 e YB42-17); da cv. Prata (ST42-08 e ST12-31) e de Prata-Anã (SH3640) e as cultivares Pacovan e Nanicão. As características utilizadas para as avaliações foram: altura da planta (m); diâmetro do pseudocaule $(\mathrm{cm})$; número de folhas vivas na floração; número de folhas vivas na colheita; número de frutos por cacho; peso do cacho $(\mathrm{kg})$; peso médio de frutos $(\mathrm{g})$; comprimento do fruto $(\mathrm{cm})$; diâmetro do fruto (cm); espessura da casca (mm); número de dias do plantio à floração e número de dias do plantio à colheita. Usou-se o delineamento em blocos casualizados com 10 tratamentos, cinco repetições e seis touceiras por parcela, espaçadas de 2,00 m x 3,00 m. Os híbridos avaliados apresentaram características agronômicas iguais ou superiores as cultivares que lhes deram origem. Os melhores híbridos de 'Prata', 'Yangambi' e 'Gros Michel' foram respectivamente, ST12-31, YB42-21 e Bucaneiro.
\end{abstract}

Termos de indexação: Musa spp., produção, genótipos, ciclo da cultura, porte da planta.

\begin{abstract}
The banana genetic breeding program at Embrapa Cassava and Tropical Fruits aims to develop and select new banana varieties with high productivity, good fruit flavor and resistance to most banana diseases. The objective of this work was to evaluate banana cultivars and hybrids in order to identify superior genotypes for productivity and disease resistance. The following characteristics were evaluated: plant height $(\mathrm{cm})$; pseudostem diameter $(\mathrm{cm})$; number of living leaves during flowering; and at harvest number of fingers per bunch; bunch weight $(\mathrm{kg})$; mean finger weight $(\mathrm{g})$; finger length $(\mathrm{cm})$; finger diameter $(\mathrm{cm})$; skin thickness $(\mathrm{mm})$; number of days from planting to flowering and number of days from planting to harvesting in the hybrids of the Gros Michel (Calipso, Bucaneiro and Ambrosia); Yangambi (YB42-21 e YB42-17); Prata (ST42-08 e ST12-31) and Prata Anã (SH3640) and in the cultivars Pacovan (Prata Type) and Nanicão (Cavendish Type). The experiment was carried out in randomized blocks design, with 10 treatments, five replications and six bushes per plot in the spacing of $2.0 \mathrm{~m}$ $\mathrm{x} 3.0 \mathrm{~m}$. The hybrids evaluated presented agronomic characteristics equal or superior to the corresponding varieties. The best hybrids of the Prata, Yangambi and Gros Michel were ST12-31, YB42-21 and Bucaneiro, respectively.
\end{abstract}

Index terms: Musa spp., production, genotypes, crop cycle, plant height.

(Recebido para publicação em 14 de Janeiro de 2004 e aprovado em 7 de março de 2005)

\section{INTRODUÇÃO}

A bananeira é cultivada em uma extensa região tropical em todo mundo, geralmente por pequenos agricultores. O Brasil é o terceiro produtor mundial, com 5,92 milhões de toneladas, em uma área cultivada de 528 mil hectares (FAO, 2003).

O desenvolvimento e seleção de novas cultivares visa, principalmente, contornar problemas fitossanitários, seja com doenças já prevalecentes na área ou com o aparecimento de novas doenças e a necessidade de reduzir o uso de agrotóxicos utilizados para o controle das mesmas (Daniels, 2000). Antes de se recomendar a substituição de uma cultivar, faz-se necessário conhecer bem o novo genótipo, mediante estudos de caracterização e avaliação em diferentes ecossistemas.

1. Engenheiro Agrônomo, M.S. Pesquisador da Embrapa Mandioca e Fruticultura - Caixa Postal 007 - $44.380-000$ - Cruz das Almas-BA mlima@cnpmf.embrapa.br

2. Engenheiro Agrônomo, D.S. Pesquisador da Embrapa Mandioca e Fruticultura - Rua Embrapa s/n - Caixa Postal 007 - $44.380-000$ - Cruz das Almas, BA.

3. Estudante de Pós-Graduação da Universidade Federal Rural de Pernambuco - Recife, PE - onildonunes@yahoo.com.br

4. Estagiária da Escola de Agronomia da Universidade Federal da Bahia - Cruz das Almas, BA.

5. Estudantes de Pós-Graduação da Escola de Agronomia - Universidade Federal da Bahia - Cruz das Almas, BA. 
Trabalhos desta natureza vêm sendo realizados em vários países, inclusive no Brasil. Um bom exemplo destes estudos, foi o trabalho em rede realizado pela INIBAP (Rede Internacional para Melhoramento de Banana e Plátano) com a participação de vários países usando genótipos selecionados em cinco programas de melhoramento de bananeira, que constatou preliminarmente, a superioridade dos híbridos da Fhia com relação ao tamanho do cacho (ORJEDA et al., 1999).

A utilização de cultivares que apresentam baixo potencial produtivo e suscetibilidade à doenças e pragas, constitui-se em uma das principais causas do baixo rendimento da bananicultura no Estado da Bahia. Uma estratégia para a solução deste problema é a criação de novas cultivares mais produtivas e resistentes à doenças e pragas, mediante o melhoramento genético, cuja etapa final do processo consiste na avaliação dos genótipos em áreas de produção (SILVA et al., 1998). As características analisadas em trabalho de tal natureza são: ciclo da cultura, altura da planta, diâmetro do pseudocaule, peso do cacho, número de frutos e comprimento do fruto (ALVES, 1990; LEDO \& AZEVEDO, 1997; SILVA \& ALVES, 1999).
Objetivou-se com o presente trabalho avaliar, em Cruz das Almas, cultivares e híbridos de bananeira visando a identificação de genótipos com características agronômicas e comerciais superiores que possam ser recomendados aos agricultores.

\section{MATERIAL E MÉTODOS}

O trabalho foi conduzido no triênio 2000/2002 na Embrapa Mandioca e Fruticultura Tropical, em Cruz das Almas, BA. Usou-se o delineamento em blocos casualizados com 10 tratamentos, cinco repetições e seis touceiras por parcela, espaçadas de 2,00 m x 3,00 m.

Foi realizada análise de variância e as médias foram comparadas pelo teste de Scott-Knott a $5 \%$ de probabilidade.

Foram avaliados os dois primeiros ciclos de produção dos híbridos de 'Gros Michel' (Calipso, Bucaneiro e Ambrosia); de 'Yangambi' (YB42-21 e YB42-17); de 'Prata' (ST42-08 e ST12-31) e de 'Prata Anã’ (SH3640) e das cultivares Pacovan, e Nanicão (Tabela 1).

TABELA 1 - Descrição dos genótipos de bananeira avaliados com Cruz das Almas, BA, 2000-2002.

\begin{tabular}{|c|c|c|c|}
\hline Genótipo & Grupo & Subgrupo & Descrição \\
\hline Pacovan & $\mathrm{AAB}$ & Prata & Cultivar mutante da Prata, de porte alto. \\
\hline Ambrosia & AAAA & - & $\begin{array}{l}\text { Híbrido de Gros Michel, porte médio, resistência à Sigatoka negra e } \\
\text { ao mal-do-Panamá. }\end{array}$ \\
\hline Bucaneiro & AAAA & - & $\begin{array}{l}\text { Híbrido de Gros Michel, porte médio, resistência à Sigatoka negra e } \\
\text { ao mal-do-Panamá. }\end{array}$ \\
\hline Calipso & AAAA & - & $\begin{array}{l}\text { Híbrido de Gros Michel, porte médio, resistência à Sigatoka negra e } \\
\text { ao mal-do-Panamá. }\end{array}$ \\
\hline Nanicão & AAA & Cavendish & Cultivar de porte médio resistente ao mal-do-Panamá \\
\hline ST12-31 & AAAB & - & Híbrido de Prata, porte alto, resistente à Sigatoka amarela. \\
\hline ST42-08 & AAAB & - & $\begin{array}{l}\text { Híbrido de Prata, resistente às Sigatokas amarela e negra e ao mal- } \\
\text { do-Panamá e com porte alto. }\end{array}$ \\
\hline YB42-21 & $\mathrm{AAAB}$ & - & $\begin{array}{l}\text { Híbrido de Yangambi, resistente à Sigatoka amarela e tolerante ao } \\
\text { mal-do-Panamá e com frutos semelhantes aos da 'Maçã'. }\end{array}$ \\
\hline YB42-17 & AAAB & - & $\begin{array}{l}\text { Híbrido Yangambi, resistente à Sigatoka amarela e tolerante ao mal- } \\
\text { do-Panamá e com frutos semelhantes aos da 'Maçã'. }\end{array}$ \\
\hline SH3640 & AAAB & - & Híbrido de Prata Anã, resistente ao mal-do-Panamá. \\
\hline
\end{tabular}

Ciênc. agrotec., Lavras, v. 29, n. 3, p. 515-520, maio/jun., 2005 
As características avaliadas foram: altura da planta (ALTP) (m); diâmetro do pseudocaule (DPCA) (cm); número de folhas vivas na floração (NFOF); número de folhas vivas na colheita (NFOC); número de frutos por cacho (NUFR); peso do cacho (PECA) (kg); peso médio de frutos (PMFR) (g); comprimento do fruto (COFR) $(\mathrm{cm})$; diâmetro do fruto (DIFR) $(\mathrm{cm})$; espessura da casca (ESCA) (mm); número de dias do plantio à floração (NDPF) e número de dias do plantio à colheita (NDPC).

\section{RESULTADOS E DISCUSSÃO}

\section{Primeiro ciclo}

$\mathrm{Na}$ Tabela 2, são apresentados os resultados das características avaliadas no primeiro ciclo, em que verifica-se que a altura de planta teve variação de $338,00 \mathrm{~cm}$ (ST12-31) a $271,00 \mathrm{~cm}$ ('Nanicão') com a formação de dois agrupamentos com base na altura ou porte da planta. No primeiro (porte alto), classificaram-se além do ST12-31, a 'Pacovan' e o ST42-48. O SH3640, outro híbrido tipo 'Prata', juntamente com os demais genótipos ficaram no agrupamento inferior (porte médio). O primeiro ciclo não é o momento apropriado para analisar o porte, pois a estabilidade só é atingida posteriormente (ALVES \& OLIVEIRA, 1999; BELALCÁZAR CARVAJAL, 1991; SOTO BALLESTERO, 1992), sendo o maior incremento na altura, normalmente, observado do primeiro para o segundo ciclo (ALVES et al., 1984).

O diâmetro de pseudocaule, variou de 23,94 $\mathrm{cm}$ (YB42-17) a 20,73 cm (cv. Pacovan). Os genótipos que apresentaram um maior diâmetro do pseudocaule são menos suscetíveis ao tombamento (SILVA et al., 1998).

$\mathrm{O}$ número de folhas vivas ou ativas no florescimento variou de 11,94 (YB42-21) a 9,62 na cultivar Pacovan, ao passo que na colheita, a cv. Pacovan apresentou maior número de folhas vivas $(7,71)$ e o híbrido SH3640, o menor número $(3,55$ folhas vivas).

Não se observou coincidência com o mesmo genótipo apresentando o maior número de folhas vivas no florescimento e na colheita.

TABELA 2 - Características de desenvolvimento de 10 genótipos de bananeira nas "épocas" do florescimento e da colheita do primeiro ciclo em Cruz das Almas BA, 2001'.

\begin{tabular}{|c|c|c|c|c|c|c|c|c|c|c|c|c|}
\hline \multirow[b]{2}{*}{ Genótipos } & \multicolumn{12}{|c|}{ Características $^{2}$} \\
\hline & $\begin{array}{l}\text { ALTP } \\
\text { (cm) }\end{array}$ & $\begin{array}{l}\text { DPCA } \\
\text { (cm) }\end{array}$ & NFOF & NFOC & NUFR & $\begin{array}{l}\text { PECA } \\
\text { (kg) }\end{array}$ & $\begin{array}{l}\text { PMFR } \\
\text { (g) }\end{array}$ & $\begin{array}{l}\text { COFR } \\
\text { (cm) }\end{array}$ & $\begin{array}{c}\text { DIFR } \\
\text { (cm) }\end{array}$ & $\begin{array}{r}\text { ESCA } \\
(\mathrm{mm})\end{array}$ & NDPF & NDPC \\
\hline ST12-31 & $338,00 \mathrm{a}$ & $23,08 \mathrm{a}$ & $11,51 \mathrm{a}$ & $4,96 \mathrm{c}$ & $108,62 b$ & $15,95 \mathrm{~b}$ & $133,55 \mathrm{a}$ & $15,39 \mathrm{c}$ & $3,54 a$ & $3,16 \mathrm{a}$ & $280,93 a$ & $411,34 a$ \\
\hline Pacovan & $329,20 \mathrm{a}$ & $20,73 b$ & $9,62 b$ & $7,71 \mathrm{a}$ & $97,52 \mathrm{c}$ & $10,91 \mathrm{c}$ & $99,82 b$ & $15,21 \mathrm{c}$ & $3,06 \mathrm{~b}$ & $3,08 \mathrm{a}$ & $258,23 a$ & $362,35 b$ \\
\hline ST42-08 & $325,60 \mathrm{a}$ & $20,92 b$ & $11,45 \mathrm{a}$ & $5,72 b$ & $87,37 \mathrm{c}$ & $12,90 \mathrm{c}$ & $141,42 a$ & $16,61 b$ & $3,48 \mathrm{a}$ & $3,44 \mathrm{a}$ & $258,55 \mathrm{a}$ & $384,63 \mathrm{~b}$ \\
\hline Ambrosia & $288,00 \mathrm{~b}$ & $22,71 \mathrm{a}$ & $10,96 \mathrm{a}$ & $6,64 b$ & $134,20 \mathrm{a}$ & $19,19 a$ & $128,00 \mathrm{a}$ & $17,19 \mathrm{~b}$ & $3,40 \mathrm{a}$ & $2,84 \mathrm{~b}$ & $270,37 a$ & $375,76 \mathrm{~b}$ \\
\hline YB42-21 & $288,00 \mathrm{~b}$ & $22,96 a$ & $11,94 \mathrm{a}$ & $6,22 \mathrm{~b}$ & $91,65 \mathrm{c}$ & $12,08 \mathrm{c}$ & $125,15 \mathrm{a}$ & $13,99 \mathrm{~d}$ & $3,76 \mathrm{a}$ & $1,76 \mathrm{c}$ & $284,55 \mathrm{a}$ & $414,68 \mathrm{a}$ \\
\hline Buccaneiro & $283,80 \mathrm{~b}$ & $22,84 a$ & $11,45 a$ & $7,32 \mathrm{a}$ & $138,13 a$ & $20,25 a$ & $134,22 a$ & $18,88 \mathrm{a}$ & $3,34 b$ & $2,56 \mathrm{~b}$ & $277,76 a$ & $388,93 \mathrm{~b}$ \\
\hline SH3640 & $278,20 \mathrm{~b}$ & $23,04 a$ & $10,17 \mathrm{~b}$ & $3,55 \mathrm{~d}$ & $108,85 b$ & $15,25 b$ & $127,84 a$ & $16,88 \mathrm{~b}$ & $3,29 b$ & $3,28 \mathrm{a}$ & $283,65 a$ & $378,64 b$ \\
\hline YB42-17 & $277,00 \mathrm{~b}$ & $23,94 a$ & $11,95 a$ & $6,06 \mathrm{~b}$ & $98,33 \mathrm{c}$ & $10,47 \mathrm{c}$ & $95,12 b$ & $13,63 \mathrm{~d}$ & $3,48 \mathrm{a}$ & $1,92 \mathrm{c}$ & $276,04 a$ & $375,75 b$ \\
\hline Calipso & $276,80 \mathrm{~b}$ & $21,61 b$ & $10,65 b$ & $7,62 a$ & $132,70 \mathrm{a}$ & $16,60 \mathrm{~b}$ & $113,27 \mathrm{~b}$ & $17,48 b$ & $3,16 \mathrm{~b}$ & $2,56 \mathrm{~b}$ & $282,14 a$ & $387,68 \mathrm{~b}$ \\
\hline Nanicão & $271,00 \mathrm{~b}$ & $21,56 \mathrm{~b}$ & $9,78 b$ & $4,74 \mathrm{c}$ & $136,00 \mathrm{a}$ & $18,89 \mathrm{a}$ & $128,20 \mathrm{a}$ & $18,56 \mathrm{a}$ & $3,38 \mathrm{a}$ & $2,88 \mathrm{~b}$ & $286,83 a$ & $384,57 \mathrm{~b}$ \\
\hline CV (\%) & 5,18 & 4,18 & 6,01 & 13,58 & 8,22 & 10,90 & 10,73 & 4,37 & 7,22 & 12,42 & 7,02 & 5,03 \\
\hline
\end{tabular}

${ }^{1}$ Médias seguidas pela mesma letra, nas colunas, pertencem ao mesmo grupo pelo teste de agrupamento de Scott \& Knott, a $5 \%$ de probabilidade.

${ }^{2}$ Altura da planta (ALTP) (m); diâmetro do pseudocaule (DPCA) (cm); número de folhas vivas na floração (NFOF); número de folhas vivas na colheita (NFOC); número de frutos (NUFR); peso do cacho (PECA) (kg); peso de pencas (PEPE) (kg); peso médio de frutos (PMFR) $(\mathrm{g})$; comprimento do fruto (COFR) $(\mathrm{cm})$; diâmetro do fruto (DIFR) $(\mathrm{cm})$; espessura da casca (ESCA) $(\mathrm{mm})$; número de dias do plantio à floração (NDPF) e número de dias do plantio à colheita (NDPC). 
As cultivares Pacovan, Calipso e Bucaneiro, foram as que apresentaram maior número de folhas vivas na colheita sendo que, no florescimento, este número foi superior a 10. Isso significa que estas cultivares apresentaram maior retenção de folhas vivas (maior tempo de vida das folhas), o que pode ser atribuído, principalmente a fatores climáticos diversos, já que as cultivares são suscetíveis à Sigatoka-amarela.

Sabe-se que o enchimento dos frutos (tamanho) está diretamente correlacionado com o número de folhas vivas na colheita. Segundo Soto Ballestero (1992), os cultivares do subgrupo Cavendish requerem o número mínimo de oito folhas ativas por planta, para o bom desenvolvimento dos frutos. Entretanto, as três cultivares que chegaram à colheita com maior número de folhas vivas (acima de sete) não foram as que produziram cachos e frutos com maior peso, exceto a cv. Bucaneiro (cacho com $20,23 \mathrm{~kg}, 138$ frutos e pesando em média $134,2 \mathrm{~g}$ ). A variação do número de folhas na colheita foi de 7,71 para 'Pacovan' a 3,55 no SH3640.

A produção avaliada mediante o peso de cachos, variou de $20,25 \mathrm{~kg}$ a $10,47 \mathrm{~kg}$, respectivamente, para os genótipos Bucaneiro e YB42-17. Os híbridos tipo 'Maçã' apresentaram produção inferior aos demais genótipos igualando-se à da 'Pacovan'. O peso médio de fruto variou de 141,42 g (ST42-08) a 95,12 g (YB42-17), enquanto o número de frutos variou de 138,12 (Bucaneiro) a 87,37 (ST42-08). Nove dos genótipos avaliados não apresentaram diferença com relação ao diâmetro dos frutos, enquanto o Calipso apresentou valor inferior para esta característica. Os valores de peso de cachos observados neste trabalho, foram em média, superiores aos obtidos por Silva et al. (2002).

Considerando-se que o peso de cacho depende do número de frutos por cacho e do peso médio dos mesmos, as cultivares com maior número de frutos/cacho e de maior peso médio, proporcionarão maior produção por cacho. Neste caso era de se esperar uma boa correlação destas duas características com a produção de cachos. No entanto, isto não foi observado, sendo que o número de frutos foi o fator que melhor explicou o peso de cachos.

Observou-se que não houve grande diferença do diâmetro do fruto dentre os genótipos avaliados. Quanto à espessura da casca, os híbridos tipo 'Prata' apresentaram casca mais espessa e os tipo 'Maçã', casca mais fina.

Verifica-se que, ao contrário do que se esperava, os agrupamentos dos genótipos quanto às características: número de dias do plantio ao florescimento e número de dias do plantio à colheita não foram semelhantes. Assim, os genótipos que apresentaram os maiores períodos do plantio à colheita, não foram os mesmos que apresentaram mais tempo para florescer, mostrando com isto que existem diferenças marcantes, entre os genótipos, no período que vai do florescimento à colheita. Vale ressaltar que os genótipos YB42-21, Calipso, Bucaneiro, Ambrosia e 'Pacovan' mantiveram posições semelhantes nos agrupamentos das duas características. O ciclo é um caráter de relevância no melhoramento genético da bananeira, já que reflete a precocidade da planta. A redução do número de dias necessários à emissão do cacho é desejada, pois representa a antecipação do retorno do investimento aplicado na lavoura (PEREIRA, 1997).

\section{Segundo ciclo}

Para altura de plantas, verifica-se que houve variação de $4.43 \mathrm{~m}$ a $2.76 \mathrm{~m}$, respectivamente para as cultivares Pacovan e Nanicão, enquanto o diâmetro do pseudocaule variou de 27,62 cm (Ambrosia) a 22,36 cm (Calipso), resultado semelhante ao observado por Silva et al. (2002). Como no primeiro ciclo, verificou-se que nem sempre os genótipos que apresentaram maior altura de planta, apresentaram maior diâmetro do pseudocaule. A altura de planta é um caráter muito importante no melhoramento da cultura e influi na densidade de plantio, manejo e consequentemente, na produção (ALVES \& OLIVEIRA, 1999). À exceção da 'Nanicão,' que manteve praticamente a mesma altura de planta, em todos os outros genótipos, observou-se um grande incremento nesta característica, do primeiro para o segundo ciclo (Tabela 3).

A exceção do ST12-31, todos os genótipos apresentaram no segundo ciclo, um menor número de folhas no florescimento e na colheita que no primeiro ciclo. As diferenças maiores ou menores observadas foram em função do genótipo em si ou do seu nível de resistência à Sigatoka amarela.

Verifica-se que o peso do cacho variou de 22,83 $\mathrm{kg}$ a $14,61 \mathrm{~kg}$, respectivamente para o genótipo Ambrosia e cv. Pacovan. No primeiro e segundo ciclos os genótipos Ambrosia e Bucaneiro apresentaram as maiores produções, enquanto que 'Pacovan' manteve-se como menos produtiva em ambos os ciclos. Vale ressaltar que o híbrido ST12-31, destacou-se como um dos melhores, com relação à esta característica. Os valores obtidos por Silva et al. (2002), para esta característica foram bastante inferiores ao deste trabalho, inclusive para o primeiro ciclo. 
TABELA 3 - Características de desenvolvimento de 10 genótipos de bananeira nas "épocas" do florescimento e da colheita do segundo ciclo em Cruz das Almas-BA, $2001^{1}$.

\begin{tabular}{|c|c|c|c|c|c|c|c|c|c|c|c|c|}
\hline \multirow[b]{2}{*}{ Genótipos } & \multicolumn{12}{|c|}{ Características $^{2}$} \\
\hline & $\begin{array}{c}\text { ALTP } \\
\text { (cm) }\end{array}$ & $\begin{array}{c}\text { DPCA } \\
\text { (cm) } \\
\end{array}$ & NFOF & NFOC & NUFR & $\begin{array}{c}\text { PECA } \\
\text { (kg) } \\
\end{array}$ & $\begin{array}{c}\text { PMFR } \\
(\mathrm{g}) \\
\end{array}$ & $\begin{array}{c}\text { COFR } \\
\text { (cm) }\end{array}$ & $\begin{array}{r}\text { DIFR } \\
(\mathbf{c m}) \\
\end{array}$ & $\begin{array}{r}\text { ESCA } \\
(\mathrm{mm}) \\
\end{array}$ & NDPF & NDPC \\
\hline Pacovan & $443,38 \mathrm{a}$ & $23,94 \mathrm{~b}$ & $8,61 \mathrm{c}$ & $2,71 \mathrm{c}$ & $92,70 \mathrm{c}$ & $14,61 b$ & $136,48 \mathrm{~b}$ & $15,85 \mathrm{~b}$ & $3,19 b$ & $3,05 \mathrm{a}$ & $546,25 b$ & $619,63 \mathrm{~b}$ \\
\hline ST42-08 & $439,03 a$ & $25,53 \mathrm{a}$ & $10,30 \mathrm{~b}$ & $4,88 \mathrm{a}$ & $88,87 \mathrm{c}$ & $17,78 \mathrm{~b}$ & $166,40 \mathrm{a}$ & $17,21 \mathbf{a}$ & $3,71 \mathrm{a}$ & $3,23 a$ & $614,13 a$ & $715,01 \mathrm{~b}$ \\
\hline ST12-31 & $431,57 \mathrm{a}$ & $27,32 a$ & $11,93 \mathrm{a}$ & $5,68 \mathrm{a}$ & $120,37 \mathrm{~b}$ & $21,88 \mathrm{a}$ & $156,61 \mathrm{a}$ & $16,10 \mathrm{~b}$ & $3,71 \mathrm{a}$ & $3,16 \mathrm{a}$ & $644,12 a$ & $806,62 a$ \\
\hline YB42-21 & $364,96 \mathrm{~b}$ & $26,38 \mathrm{a}$ & $10,17 \mathrm{~b}$ & $5,49 \mathrm{a}$ & $113,27 \mathrm{~b}$ & $16,00 \mathrm{~b}$ & $117,52 \mathrm{c}$ & $14,03 \mathrm{c}$ & $3,60 \mathrm{a}$ & $2,12 \mathrm{~b}$ & $629,85 a$ & $702,77 \mathrm{~b}$ \\
\hline YB42-17 & $357,59 \mathrm{~b}$ & $26,18 \mathrm{a}$ & $10,29 \mathrm{~b}$ & $6,02 a$ & $122,57 \mathrm{~b}$ & $16,07 \mathrm{~b}$ & $99,82 \mathrm{c}$ & $12,39 \mathrm{~d}$ & $3,24 \mathrm{~b}$ & $1,68 \mathrm{c}$ & $563,33 \mathrm{~b}$ & $676,20 \mathrm{~b}$ \\
\hline Ambrosia & $353,56 \mathrm{~b}$ & $27,62 \mathrm{a}$ & $10,65 \mathrm{~b}$ & $6,27 \mathrm{a}$ & $162,27 \mathrm{a}$ & $22,83 a$ & $127,14 \mathrm{~b}$ & $17,51 \mathrm{a}$ & $3,25 \mathrm{~b}$ & $2,72 a$ & $631,27 a$ & $716,98 \mathrm{~b}$ \\
\hline Bucaneiro & $336,27 \mathrm{~b}$ & $26,18 \mathrm{a}$ & $10,60 \mathrm{~b}$ & $5,87 \mathrm{a}$ & $159,17 \mathrm{a}$ & $22,80 \mathrm{a}$ & $134,73 \mathrm{~b}$ & $17,58 \mathrm{a}$ & $3,33 \mathrm{~b}$ & $2,36 \mathrm{~b}$ & $625,56 \mathrm{a}$ & $765,55 \mathrm{a}$ \\
\hline Calipso & $323,53 \mathrm{c}$ & $22,36 \mathrm{~b}$ & $9,83 \mathrm{~b}$ & $5,66 \mathrm{a}$ & $125,41 \mathrm{~b}$ & $20,39 a$ & $144,93 a$ & $18,22 \mathrm{a}$ & $3,36 \mathrm{~b}$ & $2,33 \mathrm{~b}$ & $605,47 a$ & $679,88 \mathrm{~b}$ \\
\hline SH3640 & $312,77 \mathrm{c}$ & $26,10 \mathrm{a}$ & $9,52 \mathrm{c}$ & $4,33 b$ & $97,65 \mathrm{c}$ & $14,62 b$ & $132,64 \mathrm{~b}$ & $15,84 \mathrm{~b}$ & $3,47 \mathrm{a}$ & $2,92 a$ & $686,64 a$ & $759,04 a$ \\
\hline Nanicão & $276,68 \mathrm{~d}$ & $22,80 \mathrm{~b}$ & $8,58 \mathrm{c}$ & $3,82 \mathrm{~b}$ & $122,76 \mathrm{~b}$ & $16,11 \mathrm{~b}$ & $116,91 \mathrm{c}$ & $17,82 \mathrm{a}$ & $3,01 \mathrm{~b}$ & $2,37 \mathrm{~b}$ & $610,80 \mathrm{a}$ & $720,52 b$ \\
\hline CV (\%) & 5,68 & 6,75 & 7,55 & 15,98 & 11,45 & 15,68 & 12,45 & 7,78 & 6,82 & 13,62 & 8,52 & 8,76 \\
\hline
\end{tabular}

${ }^{1}$ Médias seguidas pela mesma letra, nas colunas, pertencem ao mesmo grupo pelo teste de agrupamento de Scott \& Knott, a $5 \%$ de probabilidade.

${ }^{2}$ Altura da planta (ALTP) (m); diâmetro do pseudocaule (DPCA) (cm); número de folhas vivas na floração (NFOF); número de folhas vivas na colheita (NFOC); número de frutos (NUFR); peso do cacho (PECA) (kg); peso de pencas (PEPE) (kg); peso médio de frutos (PMFR) (g); comprimento do fruto (COFR) (cm); diâmetro do fruto (DIFR) $(\mathrm{cm})$; espessura da casca (ESCA) (mm); número de dias do plantio à floração (NDPF) e número de dias do plantio à colheita (NDPC).

$\mathrm{O}$ peso médio de frutos variou de $166,40 \mathrm{~g}$ para o híbrido (ST42-08) a 99,82 g (YB42-17), enquanto que o número de frutos variou de 162,27 para (Ambrosia) a 88,87 (ST42-08).

Para o número de folhas no florescimento, houve uma variação de 11,93 (ST12-31) a 8,58 para a cultivar Nanicão, que não diferiu significativamente da 'Pacovan' e do híbrido de Prata Anã SH3640.

Para diâmetro de frutos, observou-se uma pequena variação, ficando o extremo superior em 3,71 para os híbridos ST42-08 e ST12-31 e o inferior 3,01 para a cv. Nanicão. Praticamente não se observou variação na espessura da casca do primeiro para o segundo ciclo de todas os genótipos avaliados.

Para o número de dias do plantio à floração, á exceção da cv. Pacovan e do híbrido YB42-17, todos os demais permaneceram no primeiro agrupamento. Já para número de dias do plantio à colheita, houve maior variação entre os genótipos, sendo os híbridos ST12-31, SH3640 e Bucaneiro, os que apresentaram maior ciclo de produção.

\section{CONCLUSÕES}

Os híbridos avaliados apresentaram características agronômicas iguais ou superiores às cultivares que lhes deram origem. Os melhores híbridos tipo 'Prata', 'Maçã' e 'Gros Michel' foram respectivamente ST12-31, YB24-21 e Bucaneiro.

\section{REFERÊNCIAS BIBLIOGRÁFICAS}

ALVES, E. J. Principais cultivares de banana no Brasil. Revista Brasileira de Fruticultura, Cruz das Almas, v. 12, n. 3, p. 45-61, jan. 1990.

ALVES, E. J.; OLIVEIRA, M. de A. Práticas culturais. In: ALVES, E. J. (Org.). A cultura da banana: aspectos técnicos, socioeconômicos e agroindustriais. 2. ed. rev. Brasília: EMBRAPA-SPI/EMBRAPA-CNPMF, 1999. p. 335-352.

ALVES, E. J.; SHEPHERD, K.; MESQUITA, A. L. M.; CORDEIRO, Z. J. M. Caracterização e avaliação de germoplasma de banana (Musa spp.). In: CONGRESSO BRASILEIRO DE FRUTICULTURA, 7., 1984, Florianópolis. Anais... Florianópolis: Sociedade Brasileira de Fruticultura/Empasc, 1984. p. 202-212.

BELALCÁZAR CARVAJAL, S. L. El cultivo del plátano en el trópico. Cali: Feriva, 1991. 376 p. 
DANIELS, J. Que variedad de banano debo cultivar? Infomusa, Montpellier, v. 9, n. 1, p. 31-33, jun. 2000.

FAO (Roma, Itália). FAO statistical databases: agricultural production: crops primary: Brazil: bananas. Disponível em: <http://apps.fao.org/page/collections>. Acesso em: 12 abr. 2003.

LEDO, A. S.; AZEVEDO, F. F. Avaliação preliminar de genótipos de banana (Musa spp.) em Rio Branco (AC). Revista Brasileira de Fruticultra, Cruz das Almas, v. 19, n. 1, p. 51-56, abr. 1997.

ORJEDA, G.; ESCALANT, J. V.; MOORE, N. Programa Internacional de Evaluación de Musa (IMTP) fase II: sinopsis del informe final y resumen de los resultados. Infomusa, Montpellier, v. 8, n. 1, p. 3-10, jun. 1999.

PEREIRA, M. C. T. Crescimento e produção de primeiro ciclo da bananeira (Musa spp.) 'Prata-Anã (AAB) em sete espaçamentos, em Jaíba e Visconde do Rio Branco (MG). 1997. 56 f. Dissertação (Mestrado em Fitotecnia) - Universidade Federal de Viçosa, Viçosa, 1997.

SILVA, S. O.; ALVES, E. J. Melhoramento genético e novos cultivares de banana. Informe Agropecuário, Belo horizonte, v. 20, n. 196, p. 91-96, jan./fev. 1999.

SILVA, S. de O; FLORES, J. C. O.; LIMA NETO, F. P. Avaliação de cultivares e híbridos de bananeira em quatro ciclos de produção. Pesquisa Agropecuária Brasileira, Brasília, v. 37, n. 11, p. 1567-1574, nov. 2002.

SILVA, S. de O.; MATOS, A. P.; ALVES, E. J. Melhoramento genético de bananeira. Pesquisa Agropecuária Brasileira, Brasília, v. 33, n. 5, p. 693703, maio 1998.

SOTO BALlESTERO, M. Bananos: cultivo y comercialización. 2. ed. San José: Litografia e Imprenta Lil, 1992. 674 p. 